\title{
Bioética y deontología en los laboratorios de Psicología
}

JUAN PABLO PÁEZ

Fecha de recepción: 26-06-2009 - Aprobación: 03-08-2009

\section{INTRODUCCIÓN}

\section{Uso de sujetos animales en investiga- ción y educación}

Para el desarrollo profesional del psicólogo se hace necesario su ejercicio como investigador que interactúa y manipula experimentalmente con individuos y situaciones.

\section{Resumen}

En el presente artículo se revisan las principales consideraciones éticas para el desarrollo de la investigación experimental de diversa índole en organismos animales y humanos. Se ofrece, inicialmente, una justificación para el uso de sujetos animales en el laboratorio como parte de la experimentación y como herramienta educativa, y se señala la importancia de esta práctica para el desarrollo de la Psicología y otras disciplinas científicas. Se muestra también la importancia de procedimientos alternativos en la experimentación con animales y se hace énfasis en los lineamientos éticos relevantes en la formación investigativa del psicólogo. Por último, se justifica el desarrollo de la investigación experimental con participantes humanos y se señalan las consideraciones más relevantes en ese sentido, haciendo especial énfasis en los derechos como investigador que se le deben garantizar al personal incluido en la investigación y la importancia del protocolo de investigación y del consentimiento informado.

\section{Palabras claves}

Bioética, investigación animal, investigación humana, procedimientos alternativos, calidad de vida, consentimiento informado.
Por la relación generada y las implicaciones sobre el desarrollo de los organismos con los cuales se experimenta, se despierta una inquietud general por la posibilidad de presentarse desconocimiento, negligencia o maltrato hacia los sujetos experimentales en estos contextos, provocándoles, posiblemente, daños físicos y psicológicos o por la aparición de sesgos en las investigaciones y resultados incorrectos o inadecuados.

\section{Abstract}

In this paper, there is a review of the main ethic considerations for the development of the experimental investigation of different kinds on animals and humans organisms, it offers a reason for the use of animals in the lab as part of the experimentation and as an educational tool, and there is indicated the importance of this develop of the Psychology and others scientific disciplines. There is shown also the importance of alternative procedures on the experimentation with animals and there is an emphasis on the main ethic limits on the investigative growth of the Psychologist. Finally, there is explained the develop of experimental investigation with human patients and is indicated the main considerations about it, doing main emphasis on the rights that must guarantee as an investigator to the people included in the investigation, the importance of the investigation protocol and the informed assent.

\section{Keywords}

Bioethics, animal investigation, human investigation, alternative procedures, quality of life, informed assent. 
De acuerdo con el Código Deontológico y Bioético del Psicólogo, publicado en el año 2009 (artículo 2. ․ parágrafo 10), con respecto al uso y cuidado de los animales en la experimentación, la implementación y la práctica de parámetros éticos en los laboratorios de Psicología deben permitir el fortalecimiento de los investigadores y el trato adecuado con los individuos experimentales, mediante el reconocimiento de la importancia del otro en la metodología experimental y el planteamiento de posibilidades para ofrecer mayores bienestar y comodidad. Además de la obtención de resultados coherentes en las investigaciones, estas deben fomentar la formación de psicólogos integrales interesados en los fundamentos bioéticos en la práctica básica y aplicada.

Según la Organización Panamericana de la Salud (2007), la posibilidad de experimentar con animales ha permitido un mayor desarrollo de conocimientos en los diferentes campos de investigación, tanto en el bienestar del hombre como de los propios animales. Por ello, el destino de estas especies ha comenzado a despertar un gran interés. Evitar o disminuir su sufrimiento ha sido objeto de numerosos estudios en las últimas décadas, lo que ha llevado al establecimiento de reglamentaciones y, también, ha hecho pensar en las relaciones con el medio ambiente que se ve afectado.

Algunos psicólogos utilizan animales en su tarea investigativa, no solo por un interés marcado en la conducta animal, sino por la creencia de que esto puede ayudar a comprender de mejor forma el comportamiento humano; para justificar este hecho, los psicólogos han incluido la conducta, ampliando la idea de Darwin sobre la continuidad evolutiva, con el argumento de que se pueden considerar los patrones de comportamiento de los humanos en una forma más simple si observamos a las especies próximas, pero mucho menos evolucionadas (Wadeley y Blasco, 1995).

\section{Procedimientos alternativos en la expe- rimentación con animales y ética inves- tigativa}

Existen diversos campos de investigación en los que, al menos en el futuro inmediato, se necesita el empleo de animales en los experimentos. Es importante comprender que un animal vivo intacto es más que una asociación de células, tejidos u órganos. Se debe tener en cuenta, además, que ellos poseen complejas interacciones que los métodos alternativos biológicos o de otra índole no permiten duplicar, como en los casos donde se estudian fenómenos conductuales o de interacciones en el sistema nervioso. El término "alternativo" ha sido empleado para referirse a la sustitución de los animales vivos por otros procedimientos y a los métodos destinados a reducir el número de animales necesarios o a perfeccionar los procedimientos de experimentación. A partir de estos criterios, se toma el concepto de las tres $\mathrm{R}$, internacionalmente conocido, que expresa que en la experimentación animal debe preferirse toda técnica que: (a) Reemplace el uso de animales o la sustitución por invertebrados, embriones de vertebrados, microorganismos, plantas, cultivos de células, tejidos $\mathrm{u}$ órganos. (b) Reduzca la cantidad de animales empleados, sin perder la significación estadística de los resultados, logrando una menor dispersión de los datos. (c) Refine un método existente para disminuir el dolor o malestar en los animales (ops, 2007).

La legislación para ética animal, Animals (Scientific Procedures), instaurada en 1986, ha permitido orientar a todos los científicos involucrados en la investigación animal como directos responsables de mantener altos criterios éticos en su trabajo. Los psicólogos están sometidos a la legislación de 1986; por esta razón, el desconocimiento de la ley no es excusa para llevar a cabo experimentos carentes de ética, que influyan negativamente o lastimen a los sujetos experimentales. Por ello, los psicólogos pueden ser sometidos a juicio, si desarrollan su trabajo al margen de lo que establece la ley (Wadeley y Blasco, 1995).

Según el Código de Bioética y Seguridad (CBS) de 2002, en el caso de las sesiones experimentales lideradas por docentes deben eliminarse las prácticas con animales cuyos resultados forman parte del conocimiento científico aceptado. Para estos procesos educativos deben utilizarse videos y otros recursos de aprendizaje actualizados, y evitar de esta manera hacer daño innecesario; con ello también se promueve una favorable concepción ética del estudiante acerca de la responsabilidad en la investigación y se le brindan las bases para que ejerza con un adecuado proceder.

En caso tal de considerar indispensable llevar a cabo la investigación con animales porque no existe otra alternativa viable, estos deben ser de la especie, calidad y edad apropiadas a los objetivos de la misma, y su número debe constituir el mínimo necesario para alcanzar resultados válidos estadísticamente. Los investigadores y el resto del personal deben tratar en todo momento a los animales como organismos vivos sensibles, evitándoles o minimizándoles la incomo- 
didad, el sufrimiento y el dolor. Se debe considerar y prevenir el estrés provocado por el aislamiento, el miedo, la falta de espacio u otro factor capaz de afectarlos física y emocionalmente (CBS, 2002).

El investigador no debe exponer el animal a una situación que ponga en peligro su supervivencia. Los estudios no deben alterar sus procesos vitales, así como tampoco deben utilizarse animales en vía de extinción. En la investigación de laboratorio debe elegirse el diseño experimental que permita obtener el mayor nivel de observación, con la mínima molestia para los animales. Es ilegal, como ya se mencionó, causar daño o incomodidad a un animal, en el marco de una investigación (Wadeley y Blasco, 1995).

La institución de investigación o docencia en la que se utilicen animales tiene la responsabilidad de asegurar que los investigadores, profesores y el personal posean la idoneidad y experiencia para realizar determinados procedimientos. Es preciso ofrecerles adecuadas oportunidades de adiestramiento en el mismo servicio, que fomenten la responsabilidad y compasión por los animales a su cuidado. Tanto en investigación como en docencia, los procedimientos que causan dolor o sufrimiento a los animales, que no sean momentáneos o mínimos, se deben realizar después de administrar sedantes, analgésicos o anestesia, según las prácticas aceptadas en la medicina veterinaria (CBS, 2002; Colegio Colombiano de Psicólogos, 2009).

La conveniencia de la experimentación animal debe ser debatida desde las perspectivas éticas e investigativas (Wadeley y Blasco, 1995). Se hace necesario contemplar la opción de experimentación con animales, siempre y cuando se tenga la suficiente claridad por parte de los investigadores sobre cómo mantener un adecuado trato con los sujetos experimentales, así como el compromiso de ofrecerles una excelente calidad de vida, en el proceso de obtener buenos resultados y darles una aplicación correcta, correspondiente a los requerimientos de la sociedad.

Wadeley y Blasco (1995) afirman que la mayoría de los experimentos rigurosamente controlados que se han llevado a cabo con animales no hubieran sido posibles de realizar en humanos por razones éticas o legales. La investigación animal puede utilizarse para descubrir relaciones de causa y efecto en los casos donde los datos disponibles de los estudios con humanos son únicamente circunstanciales, lo que conlleva a sugerir hipótesis claras que con posterioridad puedan ser estudiadas en los humanos.

\section{JUAN PABLO PÁEZ}

Psicólogo titulado de la Universidad Católica de Colombia, magíster en Neurociencia y Biología del Comportamiento de la Universidad Pablo de Olavide, en Sevilla, España. Su interés académico se enmarca en las metodologías de investigación en Psicología comparada con humanos y no humanos, así como la pedagogía para la enseñanza de las neurociencias. Ha sido ponente en eventos nacionales e internacionales, ganador de premios y reconocimientos y autor de publicaciones en revistas nacionales. Se desempeña como director del laboratorio de Psicología del Politécnico Grancolombiano, además de impartir el curso de Neurofisiología y la electiva Comportamiento Animal.

juanpaez@poligran.edu.co

\section{Investigación experimental con seres humanos y bioética}

Los avances actuales de las ciencias de la salud se concentran en el desarrollo de la investigación humana cuando esta no implica un peligro o riesgo para los humanos; en este campo, la preocupación por el mejoramiento de la calidad de vida de ellos debe importar más que los intereses de la ciencia y de la sociedad misma. El propósito de la investigación médica en humanos es el de optimizar los procedimientos terapéuticos, diagnósticos y preventivos, además de comprender la procedencia y causa de las alteraciones relacionadas con este campo del conocimiento. Para orientar los principios éticos en el ámbito de la investigación humana, la Asociación Médica Mundial (AMM) ha promulgado la Declaración de Helsinki, la cual manifiesta como fin último del deber, la promoción y lucha por la salud, la integridad y el bienestar de las personas expuestas a cualquier clase de intervención o tratamiento en una investigación (AMM, 1964).

Toda investigación realizada con seres humanos, ya sea de carácter médico, psicológico, social, etc., debe garantizar a los individuos el respeto por su dignidad, sin discriminación de ninguna índole, al igual que la garantía de su integridad y los demás derechos con respecto a las aplicaciones de la Biología y la Medicina (Convenio de Oviedo, co, 1997). Toda interposición en el ámbito de la salud, entre las que se incluye la experimentación humana, debe realizarse respetando las normas éticas y profesionales. 
Según Hernández y Heuze (2007), la justificación ética establecida científicamente para la intervención en la investigación biomédica y psicológica con seres humanos se fundamenta en la posibilidad de descubrir nuevos beneficios para la salud y el desarrollo vital de las personas. La investigación puede justificarse solo si se realiza respetando y preservando los derechos éticos de los sujetos. En toda intervención con humanos, el investigador debe garantizar que los beneficios para el individuo y los peligros a los que se expone establezcan una relación suficientemente adecuada, en la que los riesgos hayan sido minimizados y sean menores que los beneficios; dado el caso en el que los riesgos igualen o superen los beneficios individuales, debe valorarse su importancia en el desarrollo de la sociedad (oms, 2002).

Los investigadores del campo biopsicosocial deben considerar la investigación con humanos como la última alternativa o herramienta que tenga el propósito de obtener resultados científicos que garanticen objetividad, validez y confiabilidad, garantizando manejarla solo cuando sea indispensabley estrictamente necesario y esta contribuya al desarrollo y mejora de la especie sin desmejorar sus condiciones, además de valorar de una forma segura, rigurosa, preventiva y sistemática los posibles riesgos, con el objetivo de enfrentarlos de una forma correcta y satisfactoria, sin intervenir con el desarrollo ético y moral del individuo. Es importante indicar que, si se corre algún riesgo que atente contra la integridad del sujeto, se debe suspender el experimento de inmediato (АмМ,1964).

De acuerdo con este postulado, la Organización Mundial de la Salud (oms), desde 2002, considera que al ser inevitable la aplicación experimental con seres humanos, el proyecto debe ser presentado y valorado por un comité de evaluación, científico y ético, que examine su viabilidad y validez. Antes de llevar a cabo una investigación experimental en humanos, el investigador debe obtener la aprobación y validación del comité, que, además de autorizar mediante un documento escrito, debe realizar revisiones durante el proceso de la investigación.

La responsabilidad que se adquiere con las personas que participan en una investigación es compromiso del investigador o la persona con capacitación que esté a cargo, no de los participantes, aunque hayan firmado un consentimiento informado.

\section{Del consentimiento informado}

Antes de iniciar cualquier tipo de investigación es necesaria la obtención de un consentimiento voluntario de los sujetos experimentales que participarán en ella; en el evento en que se presente incapacidad para autorizar el consentimiento, este debe ser logrado por intermedio de un representante calificado, de acuerdo con la situación específica del impedimento (oms, 2002).

El consentimiento informado, necesario para la inclusión del individuo en el desarrollo de la investigación científica, debe ofrecer la libertad de elección de la participación o no del sujeto experimental. Según el Tribunal Internacional de Núremberg (TiN), se prohíbe la influencia de fraudes o engaños, la presencia de alguna clase de presión, además del conocimiento de todas las implicaciones y alcances propios de la investigación, así como la posibilidad de retirarse de la investigación en el momento en que se desee.

Un prerrequisito para la firma del consentimiento del sujeto experimental es la información que el investigador debe proporcionar al individuo con respecto a su participación en la investigación, incluyendo principalmente la razón por la cual se considera apropiado para la investigación, la participación voluntaria y su posibilidad de negarse a continuar haciéndolo o retirarse cuando lo desee, al igual que la duración aproximada de la situación experimental, los procedimientos que se llevarán a cabo, el acceso a los resultados y datos de la investigación, la posibilidad de cualquier molestia, dolor o riesgo, los beneficios que se esperan con su participación en la investigación, la confidencialidad de los datos alcanzados, si así se requiere, y los patrocinadores o investigadores directos que colaboran en el procedimiento experimental (oms, 2002). La omisión del consentimiento informado debe considerarse inusual en todas las ocasiones; en caso tal, debe aprobarse por parte de un comité de evaluación ética (oms, 2002).

Dada la circunstancia en la que un individuo sea incapaz de otorgar su consentimiento, el investigador debe obtener la autorización de un representante considerado legal. Estos individuos deben evitarse en la investigación y ser incluidos cuando sea estrictamente necesario o, también, cuando la información alcanzada gracias a su participación sea realmente útil para el descubrimiento y mejoría de los métodos científicos y de la salud de la población, y sea obligatoria la presencia de personas legalmente incapaces porque no existe 
la posibilidad de obtener los mismos resultados con personas capaces de expresar su consentimiento. Las razones específicas por las que se utilizan en la investigación participantes que no pueden otorgar su consentimiento informado deben ser mencionadas en un protocolo experimental que se presenta para consideración y aprobación del comité de evaluación (Colegio Colombiano de Psicólogos, 2009).

Al presentarse la eventualidad en la que el sujeto está impedido para dar su consentimiento debido a un trastorno mental, este podrá ser incluido en la investigación experimental si tiene por objeto tratar dicho trastorno o cuando el tratamiento experimental sea necesario y la omisión de este perjudique su mejoría o integridad, así como de medios de elevación de recursos (Colegio Colombiano de Psicólogos, 2009).

En el caso en que la investigación a realizarse demande la participación de niños o niñas, el investigador debe justificar ante el comité de ética el porqué no puede ser realizada con adultos, circunstancia en la que es menester que el propósito de la investigación sea la obtención de conocimiento relevante sobre las necesidades de los menores. En este tipo de casos, el padre, madre o representante legal de cada niño o niña autoriza su participación sin omitir la afirmación o negación expresada por el infante, así como su deseo de continuar o dejar la investigación en la que esté incluido (oms, 2002).

Según Pascual, Frías y García (1996), cuando es necesario llevar a cabo investigaciones con mujeres en estado de embarazo, los comités de investigación, así como los propios investigadores, deben garantizar que las mujeres sean informadas de los posibles riesgos, si existen, así como los beneficios para ellas y los fetos en el momento de la investigación y en el futuro. Estas investigaciones deben realizarse si son exclusivamente necesarias y si pueden aportar información relevante en las necesidades particulares del embarazo. Debe abandonarse o replantearse, si existe la posibilidad de riesgos teratogénicos.

Cuando debido a una situación de urgencia no pueda obtenerse el consentimiento previo, podrá procederse inmediatamente después de cualquier intervención indispensable, desde el punto de vista médico, en favor de la salud de la persona afectada (Convenio de Oviedo, 1996).

Además de lograr el consentimiento por parte de los participantes, es necesario tomar las precauciones apropiadas y contar con condiciones óptimas para la protección del sujeto que ha sido vinculado a la investigación, evitando cualquier posibilidad de alteración psicológica, lesión, incapacidad física, mental y muerte. El experimento debe ser realizado exclusivamente por personas científicamente calificadas y entrenadas en estos procesos. En todas las fases se requiere la máxima precaución y capacidad técnica de quienes lo dirigen o toman parte en el mismo. Los investigadores deben prever las posibilidades de interrumpir el experimento, si se considera realmente perentorio, basándose en todas las consideraciones éticas y con la seguridad de que la continuación de la investigación atentará de una manera directa e irremediable contra los sujetos experimentales (TIN, 1947).

\section{Otros aspectos de las investigaciones en humanos}

Como se menciona en párrafos anteriores, toda investigación científica debe contar con el principio básico de protección de la vida, la salud, la intimidad y la dignidad del ser humano, de acuerdo con los principios éticos establecidos por la ley y por cada disciplina; además, debe existir un sustento teórico y bibliográfico, así como un marco empírico que sustente la necesidad y utilidad del experimento que se emprende. El método de los procedimientos experimentales en humanos debe formularse claramente en un protocolo experimental (Rebolloso, 1998), que debe enviarse para la aprobación del consejo y el comité. La investigación médica solo se justifica si existen posibilidades razonables de que la población sobre la cual se realiza la investigación pueda beneficiarse de sus resultados. Ante todas las implicaciones de la investigación, el derecho de los participantes a proteger su integridad debe ser respetado. Asimismo, es necesario tomar todas las precauciones para el bienestar de los participantes, la protección de la intimidad de los individuos y la confidencialidad de la información de los pacientes; también es indispensable la reducción de las consecuencias que atenten contra su integridad y que infrinjan sus derechos y las seguridades ofrecidas en desarrollo de la investigación. Los resultados arrojados por la investigación, que se autorizan para ser publicados, deben ser exactos, e invalidarse cualquier modificación, publicando tanto los resultados positivos como negativos (АмM,1964).

Cuando hay aplicación de test psicológicos, se recomienda el seguimiento del protocolo científico para su aplicación; de igual forma, se debe considerar el res- 
peto por los derechos de autor. En caso tal en que el test se encuentre en fase de experimentación, se debe emplear con las debidas precauciones, informando al participante el propósito, límites y resultados, como lo indican los artículos 47 y 48 del Código Deontológico y Bioético, del Colegio Colombiano de Psicólogos.

Al final de la investigación, todos los participantes deben contar con el apoyo de los investigadores en la prevención y manejo de acontecimientos inherentes a su proceso durante el procedimiento experimental. En el evento en que los tratamientos de los efectos secundarios de la investigación no existan o sean poco confiables, los investigadores deben evaluar los métodos existentes que suplan las necesidades emergentes.

La Declaración bioética de Gijón, en el año 2000, resalta que la finalidad de toda investigación con seres humanos es la de servir a la humanidad y al desarrollo de todos los países del mundo y la conservación de la naturaleza, lo cual implica que los países desarrollados que inicien y obtengan resultados de investigaciones deben compartir los beneficios con los países menos favorecidos, además de incorporar la nueva información lograda a medios por los cuales todos los individuos sean capaces de conocerla y entender los avances científicos y sus utilidades. (Declaración bioética de Gijón, 2000).

\section{Propuesta para la aplicación en el labo- ratorio}

El estudiante debe reconocer, como primera medida, todos los parámetros éticos del trabajo en el laboratorio establecidos por los diversos documentos, tratados y códigos, al igual que las implicaciones y los requisitos del trato experimental con animales, y, asimismo, las exigencias básicas para el trabajo con humanos y no humanos, diferenciando las situaciones de cada uno y la manera adecuada de aplicar los parámetros éticos.

También debe identificar las implicaciones legales por la omisión del planteamiento ético y las consecuencias próximas por la violación de los derechos de los sujetos experimentales.

Las temáticas generales inician con los aspectos éticos de las investigaciones - diferenciando los casos de sujetos experimentales humanos y no humanos -, las consideraciones previas que deben identificarse para justificar el requerimiento de la experimentación y la identificación de las utilidades y beneficios de la misma. Otra temática de gran relevancia es la observancia de la ética profesional, específicamente en Psicología.

\section{Subtemas que deben desarrollarse}

- Principios y directrices generales de la ética

- Aspectos éticos generales de la ética profesional

- Aspectos éticos de la investigación con humanos

- Aspectos éticos de la investigación con animales

- Bienestar animal

- Consentimiento

- Confidencialidad

- Protección a los participantes

- Formas adecuadas de obtención de información.

Cada uno de los subtemas propuestos sustenta la aplicación de parámetros bioéticos en el ejercicio del psicólogo en situaciones experimentales necesarias para el avance del conocimiento académico y científico.

Para la implementación de la ética en los laboratorios, es necesario que cada uno de los investigadores reconozca los procedimientos adecuados y establecidos legalmente para una investigación y el manejo que estos requieren para el desarrollo adecuado de la misma, la obtención de información eficaz y el bienestar de los sujetos experimentales. 


\section{BIBLIOGRAFÍA}

Asociación Médica Mundial. (1964). Declaración de Helsinki para las investigaciones médicas en seres humanos. Finlandia. Consultado: 15 de julio de 2007, de http://www.bioeticanet.info/documentos/Helsinki.

Colegio Colombiano de Psicólogos. (2009). Deontología y bioética del ejercicio de la Psicología en Colombia, de http://www.tribunales.colpsic.org.co/documentos/ Deontologia_libro.pdf.

Convenio de Oviedo. (1996). Convenio para la protección de los derechos humanos y la dignidad del ser humano con respecto a las aplicaciones de la Biología y la Medicina. Consultado: 10 de junio de 2007, de http://wwwbioeticanet. info/documentos/oviedo.

Hernández, J. y Heuze, Y. (2007). El programa interno para el cuidado y uso de los animales de laboratorio en las instituciones biomédicas docentes, de investigación científica e industria farmacéutica. Consultado: 25 de septiembre, de 2007, de http://www.paho.org/spanish/bio/ acta15.

Ministerio de Ciencia y Tecnología. (2002). Código de Bioética y Seguridad. Venezuela. Consultado: 25 de septiembre de 2007, de http://www.paho.org/spanish/bio/ acta15.

Organización Mundial de la Salud. (2002). Pautas éticas internacionales para la investigación y experimentación biomédica en seres humanos. Ginebra. Consultado: 5 de junio de 2007, de http://www.cioms.ch/frame_spanish_ text.htm.

Organización Panamericana de la Salud. (2007). Acta bioética número 1. Consultado: 25 de septiembre de 2007 , de http://www.paho.org/spanish/bio/acta15.

Pascual, J; Frías, M.D; García, J.F. (1996). Manual de Psicología experimental. Barcelona: Ariel.

Rebolloso, E. (1998). Evaluación de programas: ámbitos de aplicación. Barcelona: Cooperativa Universitaria San Jordi.
Sociedad Internacional de Bioética. (2000). Declaración bioética de Gijón. I Congreso Mundial de Bioética. Gijón, España. Consultado: 20 de julio de 2007, de http://www. sibi.org.

Tribunal Internacional de Núremberg. (1947). Código de Núremberg. Ética médica. Consultado: 5 de junio de 2007, de http://www.bioeticanet.info/documentos/oviedo.

Wadeley, A; y Blasco, T. (1995). La ética en la investigación y prácticas psicológicas. España: Editorial Ariel Psicología. 